\title{
Familial GATA6 mutation causing variably expressed diabetes mellitus and cardiac and renal abnormalities
}

\author{
Yang Timothy Du1, Lynette Moore ${ }^{2,3}$, Nicola K Poplawski4 and Sunita M C De Sousa1,2,4,5 \\ ${ }^{1}$ Endocrine and Metabolic Unit, Royal Adelaide Hospital, 2 School of Medicine, University of Adelaide, ${ }^{3}$ SA Pathology, \\ Women's and Children's Hospital, ${ }^{4}$ Adult Genetics Unit, Royal Adelaide Hospital, and ${ }^{5}$ Center for Cancer Biology, SA \\ Pathology and University of South Australia Alliance, Adelaide, South Australia, Australia
}

Correspondence should be addressed to Y T Du

Email

yang.du@sa.gov.au

\section{Summary}

A 26-year-old man presented with a combination of permanent neonatal diabetes due to pancreatic aplasia, complex congenital heart disease, central hypogonadism and growth hormone deficiency, structural renal abnormalities with proteinuria, umbilical hernia, neurocognitive impairment and dysmorphic features. His older brother had diabetes mellitus due to pancreatic hypoplasia, complex congenital heart disease, hypospadias and umbilical hernia. Their father had an atrial septal defect, umbilical hernia and diabetes mellitus diagnosed incidentally in adulthood on employment screening. The proband's paternal grandmother had a congenital heart defect. Genetic testing of the proband revealed a novel heterozygous missense variant (Chr18:g.19761441T>C, c.1330T>C, p.Cys444Arg) in exon 4 of GATA6, which is class 5 (pathogenic) using American College of Medical Genetics and Genomics guidelines and is likely to account for his multisystem disorder. The same variant was detected in his brother and father, but not his paternal grandmother. This novel variant of GATA6 likely occurred de novo in the father with autosomal dominant inheritance in the proband and his brother. The case is exceptional as very few families with monogenic diabetes due to GATA6 mutations have been reported to date and we describe a new link between GATA6 and renal pathology.

\section{Learning points:}

- Monogenic diabetes should be suspected in patients presenting with syndromic features, multisystem congenital disease, neonatal-onset diabetes and/or a suggestive family history.

- Recognition and identification of genetic diabetes may improve patient understanding and empowerment and allow for better tailored management.

- Identification of a genetic disorder may have important implications for family planning.

\section{Background}

GATA6 (GATA-binding protein 6) is a member of a small family of zinc finger transcription factors that is important in the regulation of cellular differentiation and organogenesis. GATA6 is expressed during embryogenesis of organs derived from the endo- and mesoderm, including the heart, pancreas, gut, lung and liver (1). Pathogenic (disease-causing) variants are associated with multisystem congenital defects, particularly cardiac defects and pancreatic agenesis/hypoplasia (1). The present case is one of only a handful of reported GATA6 kindreds with diabetes mellitus from pancreatic aplasia/ hypoplasia. It represents one of the largest kindreds, illustrating an autosomal dominant, variably expressive pattern of inheritance. Features unique to this family 
include dysmorphic facial features and proteinuria with renal biopsy findings.

\section{Case presentation}

A 26-year-old man born to non-consanguineous parents was referred to the Endocrinology Service for management of poorly controlled diabetes mellitus. He had dysmorphic features, including a long and narrow face, malar flush, reduced elbow extension, fixed flexion deformities of fourth and fifth fingers bilaterally, arachnodactyly, dolichostenomelia (height $166.5 \mathrm{~cm}$, arm span $176 \mathrm{~cm}$ ) and an umbilical hernia.

He had a complex past history:

- Pancreatic agenesis with permanent neonatal diabetes mellitus and exocrine insufficiency.

- Double outlet right ventricle, pulmonary stenosis, patent ductus arteriosus and ventricular septal defect.

- Central hypogonadism and growth hormone deficiency.

- Bifid left pelvicalyceal system, right hydrocele and right epididymal cyst.

- Unexplained proteinuria with minor focal increase in mesangial cellularity on renal biopsy (Fig. 1).

- Neurocognitive impairment on neuropsychology assessment at age 18 years.

A detailed family history (Fig. 2) revealed his older brother, IV.1, had diabetes mellitus due to pancreatic hypoplasia, truncus arteriosus, bicuspid aortic valve, hypospadias and umbilical hernia. Their deceased father, III.3, was reported to have a similar physical appearance to the proband, as well as atrial septal defect, umbilical hernia and a positive result for diabetes on employment screening, although he did not seek medical attention for this. He died at age 45 years from a myocardial infarction. Autopsy revealed severe triple-vessel disease, which in the absence of a smoking history, may have been explained by untreated diabetes; the pancreas was presumed to have undergone autolysis. The proband's deceased paternal grandmother also had an incidentally detected 'hole in the heart' and five other relatives had diabetes mellitus.

Given the striking combination of clinical features and family history, genetic testing was arranged to seek an explanation for his multisystem disorder. He indicated that the information could help his family, particularly his brother, IV.1, who had previously stated he would not have children as he did not want to pass on his pancreatic and cardiac disorders. His neonatal diabetes and positive family history were suggestive

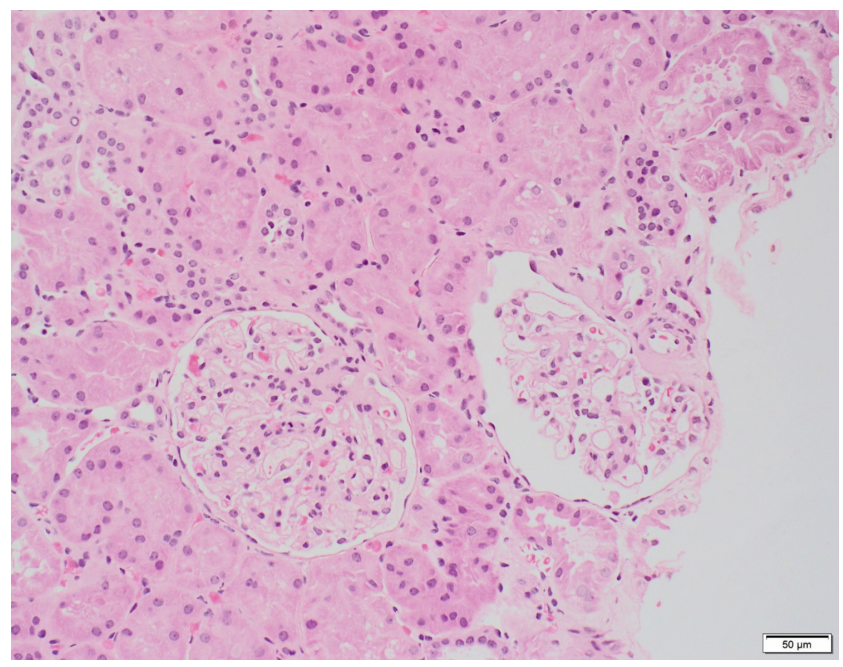

\section{Figure 1}

Proband's renal biopsy (haematoxylin and eosin (H\&E) stain, magnified 200x) showing mild focal increase in mesangial cellularity and occasional focal increase in mesangial matrix within borderline enlarged glomeruli. Ultrastructural examination by electron microscopy showed more prominent mesangial deposition but only one glomerulus was captured. Immunofluorescence examination showed only weak interactions of capillary loops with IgG and C1q. Overall, the features were not consistent with diabetic nephropathy and were instead suggestive of an immunemediated glomerulonephritis without being typical of any specific subtype.

of monogenic diabetes, whilst his concomitant heart disease and the extant literature pointed specifically to a GATA6 mutation.

\section{Investigation}

Sanger sequencing of the GATA6 gene was performed using genomic DNA extracted from peripheral leukocytes using standard procedures. This revealed a novel heterozygous missense variant (Chr18:g.19761441T >C, c.1330T $>$ C, p.Cys444Arg) in exon 4 of GATA6. This was classified as a pathogenic (class 5) variant in accordance with the American College of Medical Genetics and Genomics (ACMG) guidelines (2).

In silico modelling (Fig. 3) indicated that the change from the wild-type cysteine amino acid to the mutant arginine amino acid induced by this variant is likely to impact adversely on the function of the GATA6 protein. Arginine is a larger amino acid, which disturbs the interaction with zinc in this critical zinc finger domain of the protein, known to bind DNA. Arginine is also positively charged which causes repulsion and destabilisation of the domain. It also protrudes from the core of the protein and is unable to form the same hydrogen bond as the wildtype amino acid. 


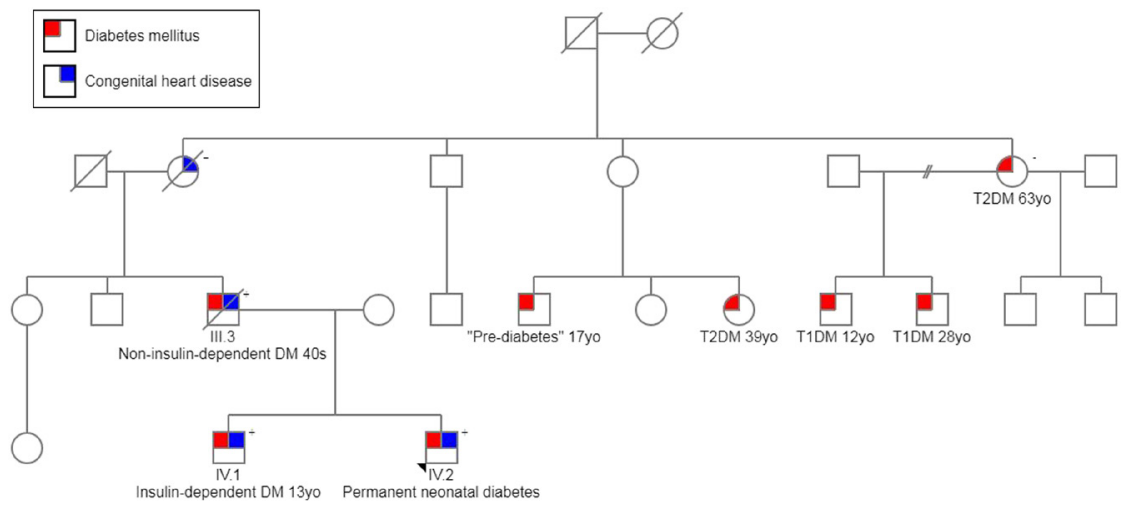

\section{Figure 2}

Pedigree showing affected family members with diabetes mellitus and/or congenital heart disease. GATA6 mutation status indicated by symbols: + mutation present; - mutation absent. DM, diabetes mellitus; TIDM, type 1 diabetes mellitus; T2DM, type 2 diabetes mellitus; yo, years old.

\section{Outcome and follow-up}

The knowledge of this genetic diagnosis has allowed the proband to better understand the aetiology of his multisystem disorder and empowered him to play a larger role in his care. Previously a frequent non-attender of clinic appointments, his therapeutic relationship with the various medical specialties involved in his care as well as adherence to treatment have improved.

Just as importantly, identification of the causative variant in the proband allowed for cascade testing of his family. Genetic testing in his brother and their parents was undertaken after genetic counselling and consent, which revealed absence of the GATA6 variant in the mother; presence of the GATA6 variant in the deceased father and presence of the GATA6 variant in the brother. It is likely that this novel GATA6 variant arose de novo in the father and was inherited in an autosomal dominant fashion in the proband and his brother. Their different clinical presentations highlight the variable expressivity of GATA6 mutations.

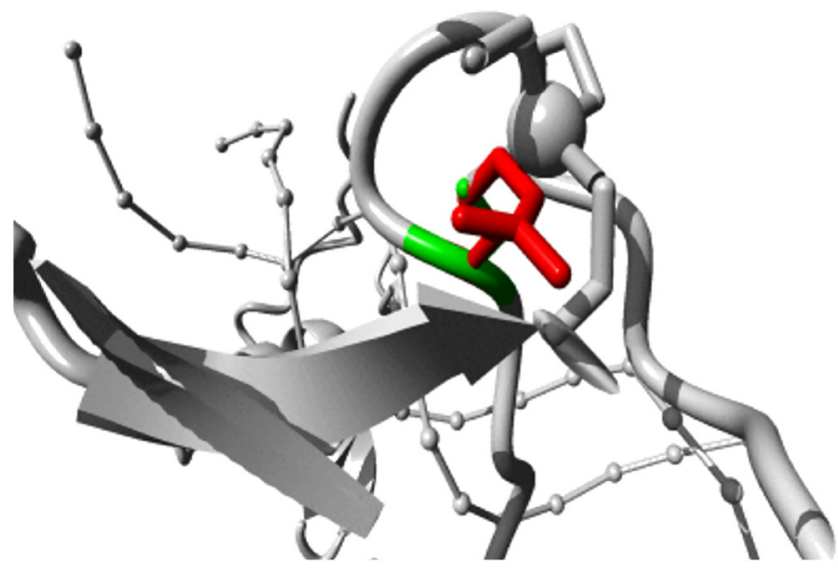

Figure 3

In silico modelling showing the GATA6 protein with the wild-type amino acid (cysteine) in green and the mutant amino acid (arginine) in red. HOPE web application used, available at http://www.cmbi.ru.nl/hope/.
IV.1, who is of normal intellect, works full time and is in a stable relationship with a long-term partner, was previously reticent about having children as he did not want to pass on his then suspected genetic multisystem disorder to his offspring. Confirmation that he inherited the familial GATA6 variant has given him the opportunity to consider reproductive technologies such as in vitro fertilisation (IVF) with preimplantation genetic diagnosis (PGD) to select for unaffected embryos and mitigate the $50 \%$ inheritance risk of this severe multisystem disorder.

\section{Discussion}

This case of neonatal-onset diabetes due to a genetic cause that was not identified until the affected proband was an adult is instructive in the general principles underlying clinical genetic testing in endocrinology.

\section{Genetic spectrum of diabetes}

Genetic forms of diabetes are often thought to be limited to the traditional forms of monogenic diabetes such as Mature Onset Diabetes of the Young (MODY); however, diabetes may also be a component of syndromic disorders due to pathogenic variants in well-defined genes. A critical clue in the proband was the neonatal onset of his diabetes. Permanent neonatal diabetes is most commonly due to mutations in KCNJ11, ABCC8, INS and GCK (3). Neonatal diabetes specifically related to pancreatic agenesis arises from mutations in multiple genes including PDX1, PTF1A, HNF1B, EIF2AK3, RFX6 and GATA6 (4). Only the latter gene is currently implicated in the combined phenotype of pancreatic agenesis and congenital heart disease (5) and enabled a successful single-gene approach in the present case using Sanger sequencing.

GATA6 was first implicated in neonatal diabetes in 2011 when heterozygous loss-of-function variants were 


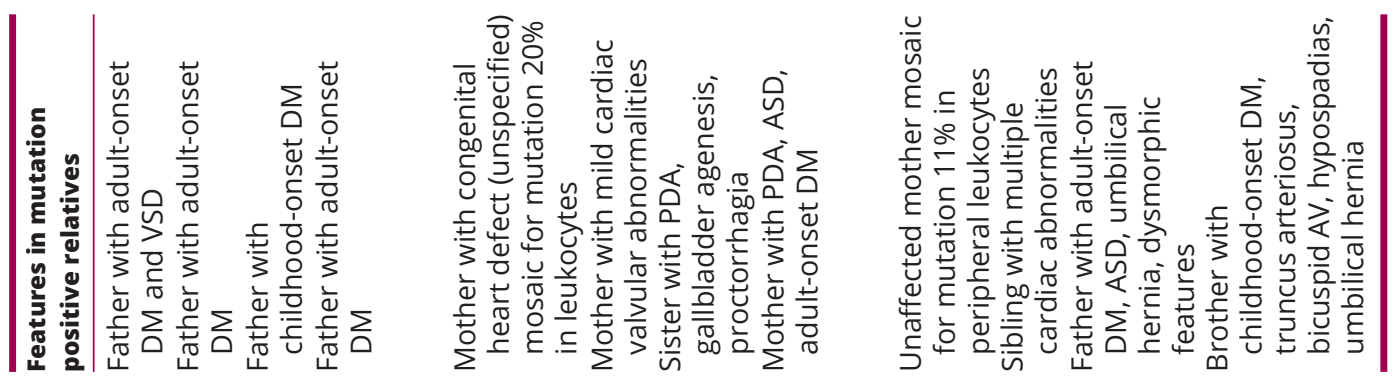

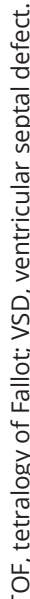
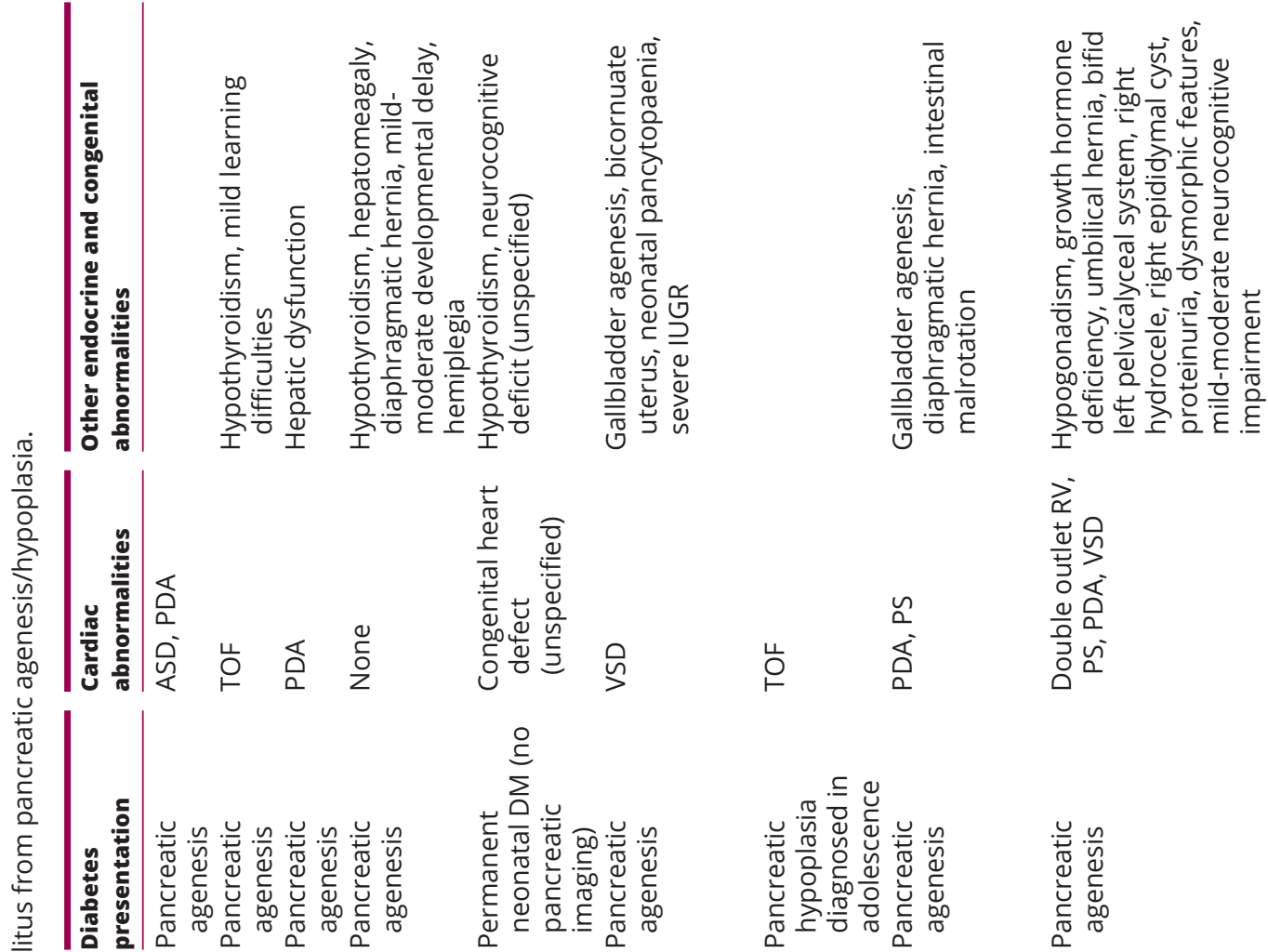

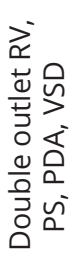
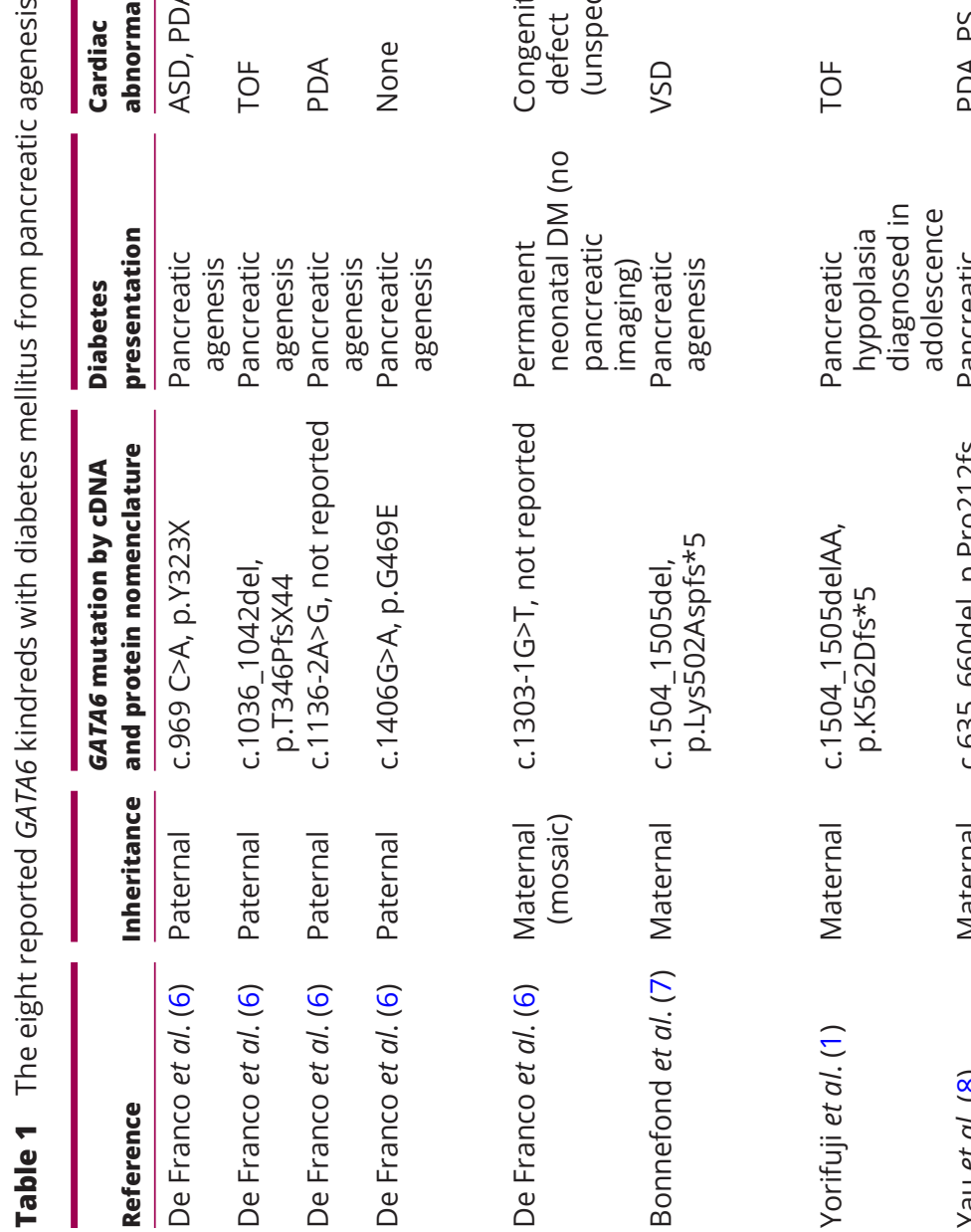

足
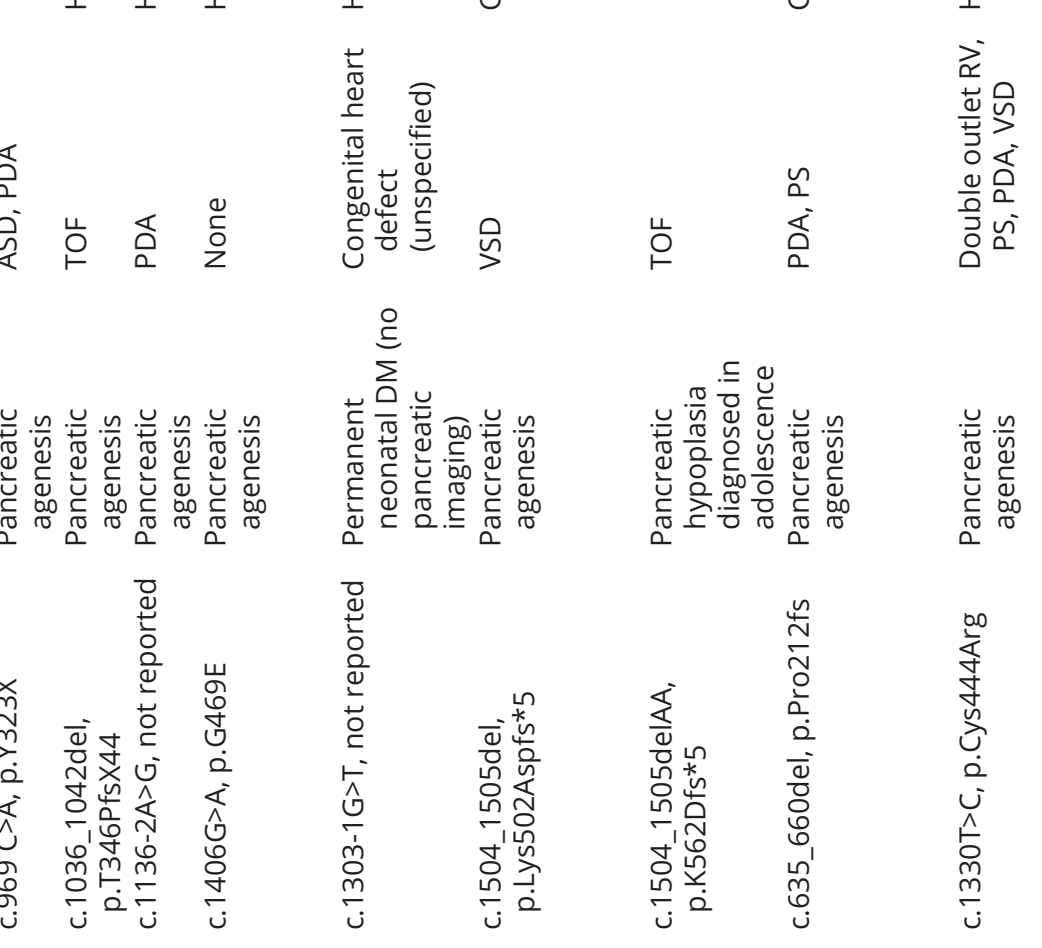

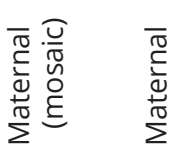
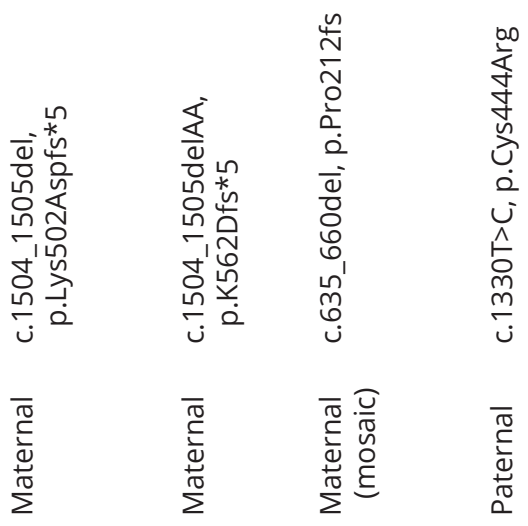

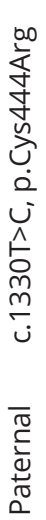

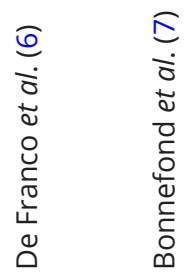

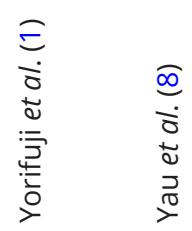

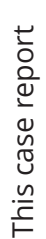


found in 15 of 27 individuals with pancreatic agenesis (5). Following multiple reports of de novo cases, only seven familial cases of GATA6-related diabetes mellitus have been previously described $(1,6,7,8)$ and the present family represents one of the largest (Table 1). The GATA6 phenotypic spectrum includes neonatal-, childhood- and adult-onset diabetes, exocrine pancreatic insufficiency, pancreatic agenesis or hypoplasia, various cardiac malformations, gallbladder agenesis/biliary atresia, hypothyroidism, hypopituitarism and pituitary agenesis, intestinal malrotation, hernias, colonic perforation, structural kidney abnormalities, neurocognitive deficits and seizures $(5,6,9)$. Other features are unique to this family. Dysmorphic facial features as observed in this case have not been described. There has been one report of proteinuria in an infant with a GATA6-truncating variant (4) and another child with a GATA6 deletion and structural renal abnormalities (9). However, to our knowledge, our case is the only report detailing renal histology in an individual with a GATA6 mutation and proteinuria. It is tempting to speculate that GATA6 may be implicated in both microscopic and macroscopic renal development. As GATA6 overexpression in rat glomerular mesangial cells results in cell cycle arrest (10), loss-of-function GATA6 variants could plausibly result in the mesangial cell proliferation observed on the proband's renal biopsy.

\section{Approach to genetic testing}

Recognising a potential genetic disorder and accurately diagnosing it through the appropriate genetic test may pay dividends across the healthcare of an individual and their family. Whether genetic testing should be pursued should reflect how likely a disorder is to be genetic and how likely a genetic result would be to alter management of the individual and/or their family. It should only be performed after comprehensive phenotyping using history, examination and preliminary investigations to determine the likelihood of finding a genetic disorder and identify candidate genes.

It is impossible to know all genetic disorders within endocrinology, but features such as young-onset and/or aggressive disease or the development of multiple rare disorders within an individual or one or more related rare disorders within multiple family members is suggestive of a genetic cause. A strong autosomal dominant family history of sulphonylurea-sensitive diabetes mellitus in lean family members with glycosuria immediately brings to mind monogenic diabetes due to a mutation in HNF1A. Monogenic diabetes due to GATA6 mutations are much rarer but the phenotype of pancreatic agenesis, congenital heart disease and other congenital malformations should raise the suspicion of some sort of genetic disorder worthy of further evaluation.

Though next-generation sequencing (NGS) may be the modality typically used to evaluate monogenic diabetes, GATA6 is one of the special cases where Sanger sequencing is preferable. GATA6 contains a $\sim 300 \mathrm{bp}$ region with high guanine-cytosine content, which is difficult to amplify because of its high stability with formation of secondary structures and higher melting points, and Sanger sequencing using tailored PCR settings can circumvent this issue.

\section{Utility of genetic testing}

The indications for genetic testing in endocrinology include differentiation of clinical disorders; disease monitoring; therapeutic guidance; prognostication and predictive testing of family members. In our case, though the genetic test results did not directly lead to a change in medical treatment for the proband, the knowledge of the diagnosis empowered the patient to take a greater part in his care and improved adherence. In addition, genetic testing with successful identification of the culprit variant allowed for cascade testing and family planning. Even though IV.1 was previously reticent about having children due to the risk of passing on his multisystem disorder, the identification of his genetic condition has given him the opportunity to consider reproductive technologies that would not have been available without the genetic test result.

\section{Declaration of interest}

The authors declare that there is no conflict of interest that could be perceived as prejudicing the impartiality of the research reported.

\section{Funding}

This research did not receive any specific grant from any funding agency in the public, commercial or not-for-profit sector.

\section{Patient consent}

Written informed consent has been obtained from the patient for publication of this article.

\section{Author contribution statement}

Y T D and S M C D were involved in the conception, literature review and drafting of this case report. S M C D arranged genetic testing of the 
proband and relatives and performed in silico protein modelling. L M was responsible for the renal biopsy histopathological assessment. $\mathrm{N} \mathrm{K}$ oversaw genetic testing of the family. All authors contributed to the final manuscript.

\section{Acknowledgements}

The authors thank the University of Exeter Molecular Genetics Laboratory for performing GATA6 Sanger sequencing.

\section{References}

1 Yorifuji T, Kawakita R, Hosokawa Y, Fujimaru R, Yamaguchi E \& Tamagawa N. Dominantly inherited diabetes mellitus caused by GATA6 haploinsufficiency: variable intrafamilial presentation. Journal of Medical Genetics 201249 642-643. (https://doi.org/10.1136/ jmedgenet-2012-101161)

2 Richards S, Aziz N, Bale S, Bick D, Das S, Gastier-Foster J, Grody WW, Hegde M, Lyon E, Spector E, et al. Standards and guidelines for the interpretation of sequence variants: a joint consensus recommendation of the American College of Medical Genetics and Genomics and the Association for Molecular Pathology. Genetics in Medicine 201517 405-424. (https://doi.org/10.1038/gim.2015.30)

3 Naylor RN, Greeley SA, Bell GI \& Philipson LH. Genetics and pathophysiology of neonatal diabetes mellitus. Journal of Diabetes Investigation 20112 158-169. (https://doi.org/10.1111/j.20401124.2011.00106.x)

4 Tuhan H, Catli G, Anik A, Ozmen D, Turkmen MA, Bober E \& Abaci A. Neonatal diabetes mellitus due to a novel mutation in the GATA6 gene accompanying renal dysfunction: a case report.
American Journal of Medical Genetics: Part A 2015 167A 925-927. (https://doi.org/10.1002/ajmg.a.36984)

5 Allen HL, Flanagan SE, Shaw-Smith C, De Franco E, Akerman I, Caswell R, International Pancreatic Agenesis Consortium, Ferrer J, Hattersley AT \& Ellard S. GATA6 haploinsufficiency causes pancreatic agenesis in humans. Nature Genetics 201144 20-22. (doi:10.1038/ ng.1035)

6 De Franco E, Shaw-Smith C, Flanagan SE, Shepherd MH, International NDM Consortium, Hattersley AT \& Ellard S. GATA6 mutations cause a broad phenotypic spectrum of diabetes from pancreatic agenesis to adult-onset diabetes without exocrine insufficiency. Diabetes 201362 993-997. (https://doi.org/10.2337/ db12-0885)

7 Bonnefond A, Sand O, Guerin B, Durand E, De Graeve F, Huyvaert M, Rachdi L, Kerr-Conte J, Pattou F, Vaxillaire M, et al. GATA6 inactivating mutations are associated with heart defects and, inconsistently, with pancreatic agenesis and diabetes. Diabetologia 201255 2845-2847. (https://doi.org/10.1007/s00125-012-2645-7)

8 Yau D, De Franco E, Flanagan SE, Ellard S, Blumenkrantz M \& Mitchell JJ. Case report: maternal mosaicism resulting in inheritance of a novel GATA6 mutation causing pancreatic agenesis and neonatal diabetes mellitus. Diagnostic Pathology 201712 1. (https://doi. org/10.1186/s13000-016-0592-1)

9 Bui PH, Dorrani N, Wong D, Perens G, Dipple KM \& QuinteroRivera F. First report of a de novo 18q11.2 microdeletion including GATA6 associated with complex congenital heart disease and renal abnormalities. American Journal of Medical Genetics: Part A 2013 161A 1773-1778. (https://doi.org/10.1002/ajmg.a.35974)

10 Nagata D, Suzuki E, Nishimatsu H, Yoshizumi M, Mano T, Walsh K, Sata M, Kakoki M, Goto A, Omata M, et al. Cyclin A downregulation and p21(cip1) upregulation correlate with GATA-6-induced growth arrest in glomerular mesangial cells. Circulation Research $20008 \mathbf{8}$ 699-704. (https://doi.org/10.1161/01.RES.87.8.699) 